\title{
Flexible Configuration of Wireless Sensor Network for Monitoring of Rainfall-Induced Landslide
}

\author{
Gian Quoc, Anh', Duc-Tan, Tran², Nguyen, Dinh Chinh³, Tien Bui, Dieu ${ }^{4}$ \\ 1,2,3Faculty of Electronics and Telecommunication, VNU, Hanoi - University of Engineering and Technology, 144 Xuan \\ Thuy, Cau Giay, Hanoi, Vietnam \\ ${ }^{1}$ Department of Electronics, Nam Dinh University of Technology Education, Phu Nghia, Loc Ha, Nam Dinh, Vietnam \\ ${ }^{4}$ Geographic Information System group, Department of Business and IT, University College of Southeast Norway, \\ Gullbringvegen 36, B $\emptyset$ i Telemark N-3800, Norway
}

\begin{tabular}{l} 
Article Info \\
\hline Article history: \\
Received May 21, 2018 \\
Revised Jul 12, 2018 \\
Accepted Oct 12, 2018 \\
\hline Keywords: \\
Landslides \\
Wireless Sensor Network \\
Early Warning \\
Topology \\
Vietnam
\end{tabular}

\section{Corresponding Author:}

Gian Quoc, Anh,

Faculty of Electronics and Telecommunication,

VNU, Hanoi - University of Engineering and Technology,

144 Xuan Thuy, Cau Giay, Hanoi, Vietnam,

Email: gianquocanh@gmail.com

\begin{abstract}
Establishment of wireless sensor network for Monitoring and Early Warning System (MEWS) of rainfall-induced landslide is still a challenging task due to problems of energy consumption. This research aim is to propose a new flexible system for the configuration of MEWS for rainfall-induced landslides. The proposed system is an integration a star topology and a tree topology, in which, the star topology is automatically used for the sensor nodes around the gateway node, whereas the tree topology is adopted for the other nodes. Consequently, the proposed configuration method is flexible and capable to save the energy consumption in the MEWS. Experiment result showed that the performance of the MEWS with the proposed method Wireless Sensor Network is better than those from the systems only use the tree topology or the star topology. The result of this study is useful for designing WSN for MEWS of rainfall-induced landslides.
\end{abstract}

Copyright $(\odot) 2018$ Institute of Advanced Engineering and Science. All rights reserved.

\section{INTRODUCTION}

Monitoring and Early Warning System (MEWS) for rainfall-induced landslide is an efficient tool for landslide management and its risk reduction. Basically, a MEWS for landslide consists of several components such as (1) sensor networks for monitoring parameters of the landslide; (2) telecommunication system; (3) monitoring and analysis station; (4) decision making; and (5) response procedures. However, design and implementation of landslide MEWS are not easy tasks because they are dependent on various factors. One of these factors is the telecommunication system that uses to send timely the monitoring parameters of the landslide to the station for further analyzing and making decision.

An effective telecommunication system could be derived with the use of a relative new technology of Wireless Sensor Network (WSN) that has proven viable, reliability, flexibility and easy for extending in some recent works [1-4]. WSN is also suitable for MEWS established in harsh environments because sensor nodes use its internal battery. The main disadvantage of WSN is that it uses low bandwidth and short range communication. Moreover, WSN exists limitations of processing capability and storage of individual sensor nodes [5]. However, if WSN is used for a long-term MEWS of landslide, dissipation of energy in the sensor nodes, microcontroller units, and transceivers of WSN is a critical issue. Thus optimization of the energy consumption for WSN is still needed better solutions. 
Literature review shows that various solutions for reducing energy consumption and extending the WSN lifetime have been proposed i.e. data compression [6, 7], low energy architecture sensor nodes [8, 9], routing protocols [10], layer optimization and sampling rate changing [11]. In general, these provide better dynamic tradeoff solutions among performance; communicate range, and network's lifetime. Nevertheless, optimization of the energy consumption in sensor nodes of WSN is still critical because the dispensed energy is proportional to the square of the distance from the sensor nodes to the monitoring and analysis station. For example, a battery $6600 \mathrm{~mA}$ only uses for 7.6 days for their sensor nodes in active mode [11], and this is clear not sufficient for long time MEWS.

This paper addresses the aforementioned critical issue by proposing a new flexible method for the configuration of MEWS for rainfall-induced landslides aiming to extend the life time of the MEWS system. The proposed configuration is an integration of the star topology and the tree topology that enable flexible scenarios. Accordingly, when a landslide is monitored and analyzed its instability by mean of Factor of Safety (FoS), the tree topology is used if FoS larger than 1, called normal condition. For this case, sensor nodes of WSN near the gateway switch to router mode to save the energy consumption. On contrast, the star topology will be automatically used, and in this case, sensor nodes far from the gateway in the star topology will consume much energy, but the network is reliable. Because, every sensor node will send and receive data directly to the gateway, therefore, the operation of a sensor node does not depend on other nodes.

\section{BACKGROUND OF WIRELESS SENSOR NETWORK AND ITS TOPOLOGY}

This section describes briefly on Wireless Sensor Network (WSN), Tree Topology and Star topology uses in MEWS for rainfall-induced landslide.

\subsection{Wireless Sensor Network}

A WSN could be defined as a set of sensed devices (also called sensor nodes) that is spatially distributed in the rainfall-induced landslide being considered, in which, sensors in these nodes could sense parameters of the landslide (i.e. pore-water in slope soil, tilt and vibration) and wirelessly communicate the obtained parameter values via wireless links. The data values are then transferred to a sink to connect to the internet or other networks through a gateway [12].

WSN has been success fully applied for various real-world problems such as food and agriculture monitoring [13], flash-flood alerting [14], soil moisture monitoring [15], civil structures monitoring [16], underground structure monitoring [17], and underground coal mine monitoring [18]. However, few researches on application of WSN for landslide study have been carried out i.e. landslide detection [19], landslide prediction [20], and landslide early warning system [21, 22]. Therefore, research on WSN for landslide MEWS should be still carried out.

\subsection{Topology of Wireless Sensor Network}

Designation of an effective landslide MEWS requires determining an appropriate topology for WSN. This is a critical importance to ensure both the reliability and the energy conservation of WSN [23]. Literature review shows that various types of topology for WSN have been used i.e. underground topology and hybrid topology [23], linear topology [24], mesh network topology and peer-to peer- topology [12], star topology [25], and tree topology [16]. Different topologies were proposed because obstructions in the environment of realworld problems may limit or prevent communication between some nodes. In this section, the star topology and the tree topology as shown in Figure 1 are shortly discussed.

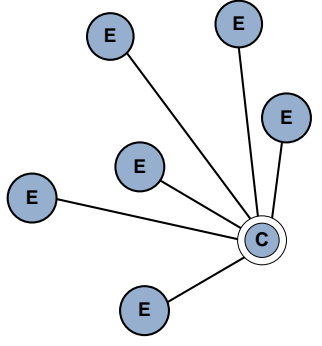

a) Star Topology

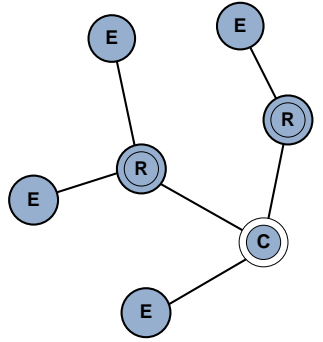

b) Tree Topology

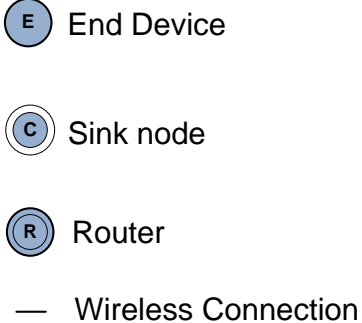

- Wireless Connection

Figure 1. Network topology: (a) Star topology and (b) Tree topology 
For the star topology, the coordinator (sink node) in Figure 1(a) acts as the network controller and the other devices are called "end-devices". The end-devices do not communicate directly with each other but operate independently. More importantly, they are not affected by other end-devices when these devices do not operate. When the star topology is active, the end-devices communicate directly with the coordinator (sink node).

In addition, an end-device may switch to the idle mode or the sleep mode in order to reduce the power consumption and extend the duration of the operation time of the sensor node. Because the end-devices do not communicate through the routers to the coordinator, the more distance between the end-device and the coordinator, the more energy spending is. The star topology should be used for landslide monitoring when the landslide is active status.

Regarding to the tree topology in Figure 1(b), the network consists of one coordinator, several routers, and end-devices. The coordinator sets up the network, selects the operating channel, give addresses for the routers and the end devices. The routers communicate directly with the coordinator. In the tree topology, the routers transfer the data from the end-devices to the coordinator, therefore, the routers are always active that consume energy. However, the distance from the end-devices to the routers is shorter than those from the enddevices to the coordinator of the star topology (Figure 1), therefore, the WSN system using the tree topology consumes less energy than that of the star topology. Thus, the tree topology should be used when the landslide is quite stable status.

\section{EXPERIMENTS AND RESULT}

\subsection{Designation the Wireless Sensor Network System for Rainfall-induced Landslide}

In this research, the Wireless Sensor Network (WSN) system was designed with 6 sensor nodes as shown in Figure 2 placed in positions on a slope surface established in the sensor lab of VNU University of Engineering and Technology (Vietnam). The sink node was placed near the slope at a safe distance. A weather station, which connects directly to the sink node, provides monitoring data of rainfall and its intensity and duration. The sensor nodes near the sink node can switch between the two functions, the router and the enddevice. Besides the battery power, the sensor nodes operating as the routers are also powered by solar panels. The sensor nodes that are far from sink node are set up as the end-devices.

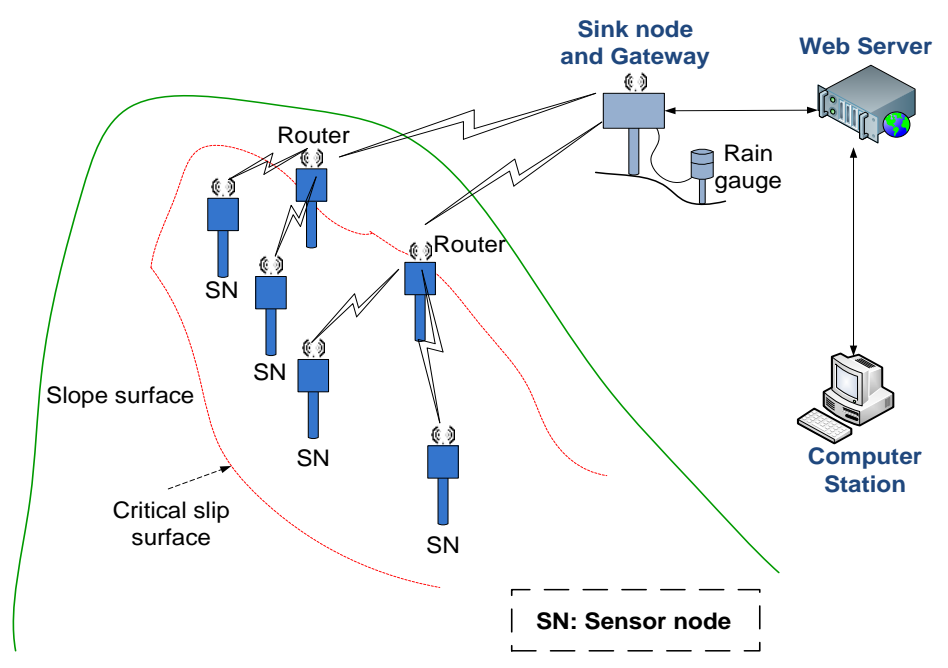

Figure 2. The network of sensor nodes used in this research

It is noted that these end-devices can communicate to the coordinator (the star topology) or through the routers (the tree topology). Data is transferred to the sink node for further processing. A gateway connects the WSN to the internet to expand the ability of communication. A computer station uses the field survey data and real-time data acquired from sensor nodes for analyzing the status of the slope.

\subsection{Designation of the Sensor Nodes for Rainfall-induced Landslide}

Block diagram of a sensor node is shown in Figure 3a, including sensors, Waspmot board, rechargeable battery, XBee-Pro ZigBee RF module. Sensors should be selected based on the degree of 
accuracy, the lifetime of operation, and power consumption [23]. Therefore, in this research, three sensors, a soil moisture sensor, a temperature sensor, and an acceleration sensor were used. Accordingly, Waspmote board, which is powered by a battery and a solar panel, is used for the sensor nodes to reduce energy consuming

Because the operation time of the Waspmote board using battery only can be 1 to 5 years depending on rate of communication used, therefore, in this study, a Waspmote PRO v1.2 is employed and designed with an ATmega1281 microcontroller (frequency 14MHz, SRAM 8KB, Flash 128KB), whereas XBee is used for wirelessly communication. We use a rechargeable battery $(6600 \mathrm{mAh}$ and $3.7 \mathrm{~V})$ for the sensor nodes. For those acts as the routers, the extra solar panel is employed.
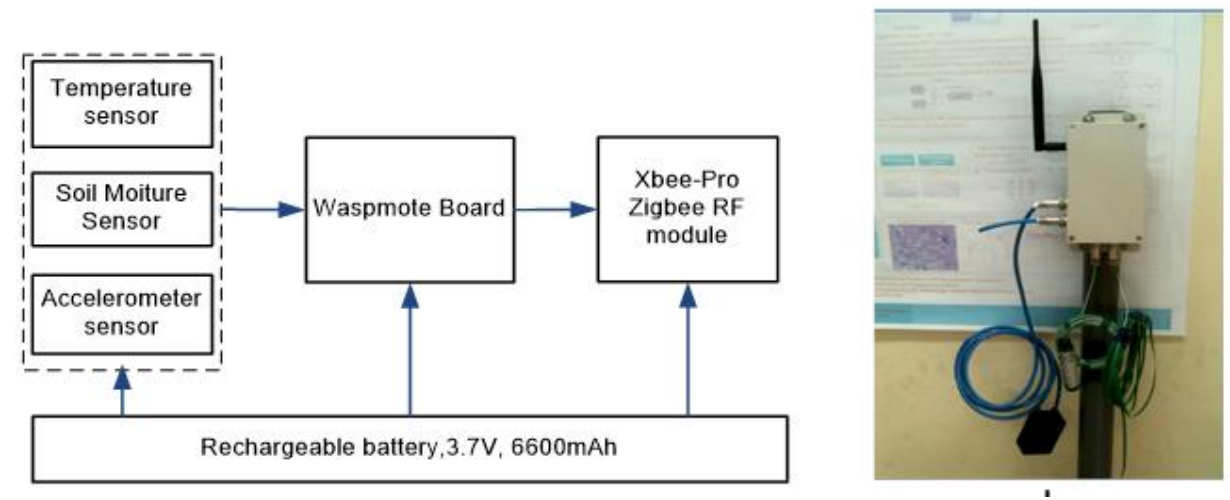

Figure 3. (a) Block diagram of a sensor node; (b) a photo of a real sensor node

Regarding the ZigBee network, we select the ZigBee for landslide monitoring in this study due to low power consumption. This is an RF protocol, in which, two lower layers MAC (Media Access Control) and Physical layer defined by IEEE 802.15.4 were used. ZigBee is targeted for using in WSN with low power, low bandwidth, and short communication range. The ZigBee network includes a coordinator, routers and end devices. The coordinator chooses PAN ID and operating channel, generates addresses to routers and end devices.

In this work, the coordinator acts as the sink node, where the routers join the network components, transmit and receive the monitoring data to the coordinator and transfer data from other devices to the coordinator. The end-devices are sensor nodes that monitor the parameters and transfer to the coordinator.

\subsection{Working Principle of the WSN System}

The sensor nodes sense the slope parameters and transfer the obtained data to the sink node, then, the gateway uploads this data to the web database via the internet. The data is analyzed at the station using the GeoStudio software to assess the status of the slope, stable or unstable, in term of Factor of Safety (FoS) as shown in Figure 4. It is noted that based on the FoS values, the system could be switched between the tree topology and the star topology.

In this study, we divide the operation of the system into two scenarios: 1) normal operation mode; 2) warning operation mode. The first scenario is a safe condition when the weather is good, pore water pressure is low and the slope vibrates below a threshold. The second scenario is an unsafe condition when the weather is bad, pore water pressure is high and the slope vibrates over a threshold.

In the normal operation mode, FoS is much greater than one, the network uses the tree topology. Whereas in the warning operation mode, slope stability is low or vibrates over a threshold, the priority of the system is data collection; therefore, the high sample rate and transfer rate are used to monitor the slope's condition. If FoS increases, the slope comes back the safe state, the network switch from the star topology to the tree topology. Accordingly, the sensor nodes turn into the idle mode to save energy. The power for the sensors is turned off, and the Waspmote board and the RF module also switch to the idle mode. 


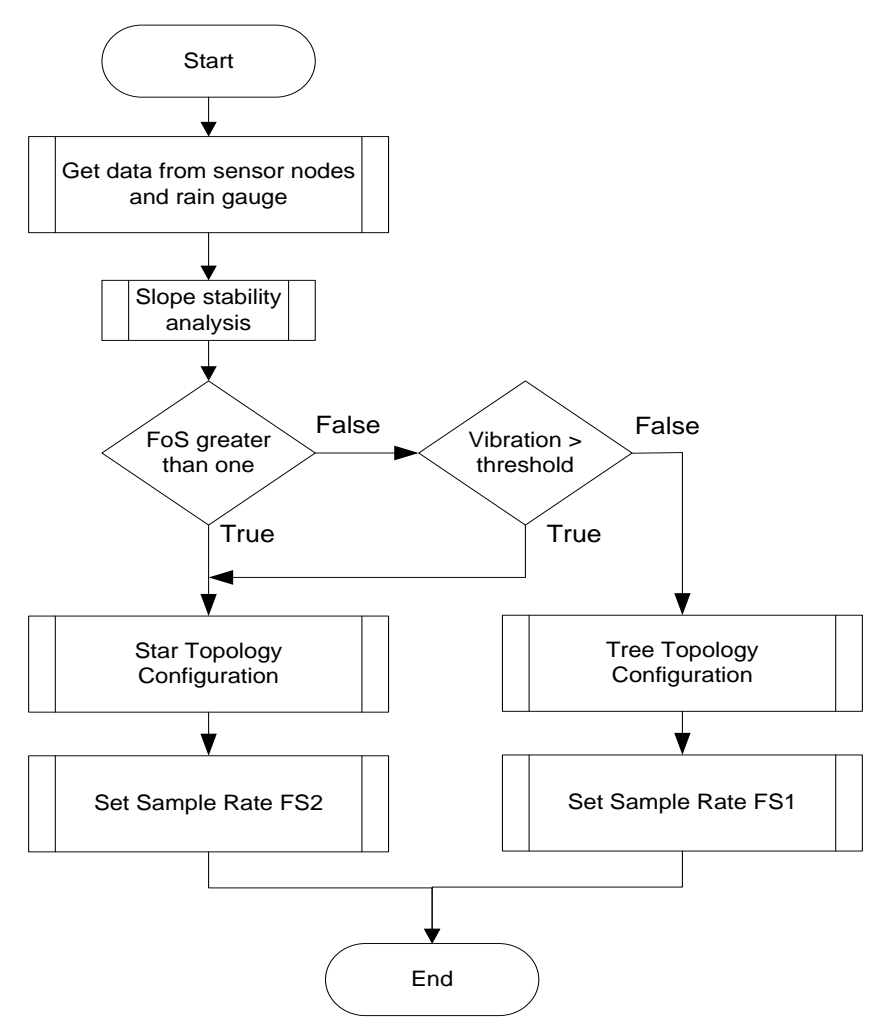

Figure 4. Working principle of the proposed system

\subsection{Results and Discussion}

The result of the estimation of the power consumption is shown in Table 1. It could be seen that the power a sensor node consumed in the active mode is $100.255 \mathrm{~mW}$. For the battery with the capacity of $6600 \mathrm{mAh}$ and voltage of $3.7 \mathrm{~V}$, the maximum power supply for a sensor node is $24420 \mathrm{mWh}$. Therefore, the working time of the sensor node may extend roughly to 243.5 hours.

In fact, the efficiency of the battery is much smaller. For example, if the efficiency is $75 \%$, the working time decreases to 182.6 hours. This working time is not enough for the long time monitoring system of landslide. In order to extend the lifetime of the network, the sensor nodes should only be active in limited time and turned to the idle mode.

Table 1 . The power consumed by a sensor node

\begin{tabular}{ccc}
\hline Module & Idle mode & Power $(\mathrm{mW})$ \\
& 0.18 & Active mode \\
\hline Waspmote & & 49.5 \\
XBee-PRO ZigBee & & $10 \mathrm{~mW}(+10 \mathrm{dBm})$ for International variant \\
Accelerometer & & 1.155 \\
Soil moisture sensor & 6.6 \\
Temperature sensor (LM35) & & 33 \\
\hline
\end{tabular}

In the normal operation mode, the end-devices only active 3 seconds after staying 10 minutes in the idle mode. The power the sensor node uses is roughly $0.656 \mathrm{mAh}$. Therefore, the lifetime of the sensor node is 1163 days considering the efficiency is $75 \%$. In warning operation mode, the sensor node active 3 seconds after staying 1 minute in the idle mode. The lifetime of the sensor node is 161 days considering the efficiency is $75 \%$.

The above result is clearly better than in the previous works in [12] in term of the power consumption. In [12], the network is switched from the tree topology to the star topology when the rainfall reaches to the determined threshold. It happens even though the FoS is still high (i.e. FoS >>1). In our work, the WSN system is switched from the tree topology to the star topology only when FoS reaches to 1. Thus, it can save more power. 
One of the most critical issues of the WSN is the transmission reliability. In this research, the transmission reliability of the proposed WSN system is assessed using Packet Delivery Ratio (PDR) measure. This is the ratio between the number of messages that the sensor nodes transmitted and the number of messages the sink node received. The result is shown Tables 2 and 3 and Figure 5. It could be seen that the total number of packets the sensor nodes sent is 730 , whereas the total number of packets the sink node received is 730 for both the star topology and the tree topology, therefore, the PDR parameter is $100 \%$.

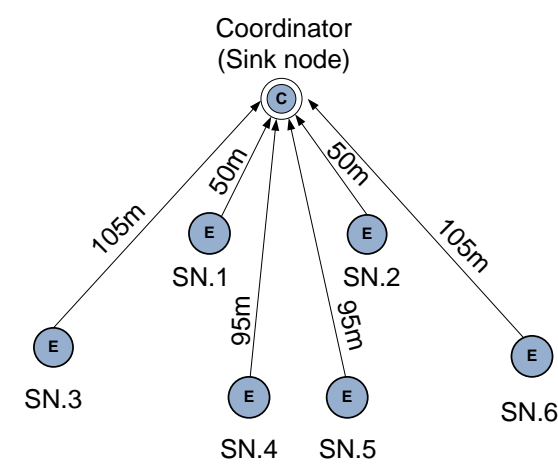

a) Star Topology

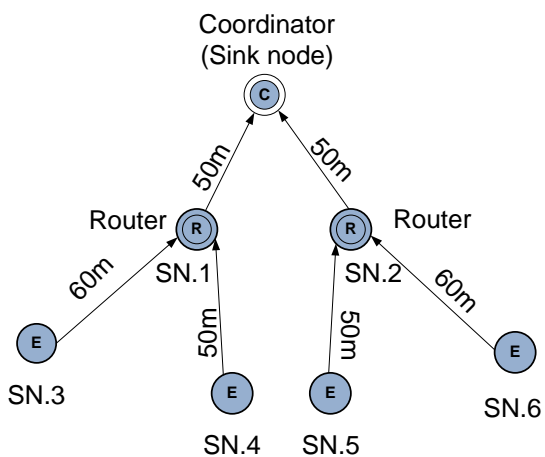

b) Tree Topology

Figure 5. Scenarios for outdoor experiment; (a) star topology; (b) tree topology

Table 2. Packet Delivery Ratio (PDR) for the tree topology

\begin{tabular}{cccc}
\hline Node & Total of transmitted packets & Total of received packets & Percent of received packets \\
\hline 1 & 140 & 140 & $100 \%$ \\
2 & 103 & 103 & $100 \%$ \\
3 & 127 & 127 & $100 \%$ \\
4 & 109 & 109 & $100 \%$ \\
5 & 133 & 133 & $100 \%$ \\
6 & 118 & 118 & $100 \%$ \\
\hline
\end{tabular}

Table 3. Packet Delivery Ratio (PDR) for the star topology

\begin{tabular}{cccc}
\hline Node & Total of transmitted packets & Total of received packets & Percent of received packets \\
\hline 1 & 140 & 140 & $100 \%$ \\
2 & 103 & 103 & $100 \%$ \\
3 & 127 & 127 & $100 \%$ \\
4 & 109 & 109 & $100 \%$ \\
5 & 133 & 133 & $100 \%$ \\
6 & 118 & 118 & $100 \%$ \\
\hline
\end{tabular}

\section{CONCLUSION}

This research has proposed a new flexible system for the configuration of MEWS for rainfall-induced landslides. The proposed system is a combination of the star topology and the tree topology to use in different scenarios of monitoring that is not only to save energy and but also to improve operational reliability of the MEWS system. The star topology is used when the movement rate of the landslide is high $(\mathrm{FoS} \leq 1)$, whereas the tree topology is employed when the slope is stable $(\mathrm{FoS}>1)$.

Experiment result showed that the performance of the MEWS with the proposed method WSN is better than those from the conventional systems using only the tree topology or the star topology. The result of this study is useful for designing wireless sensor networks for MEWS of rainfall-induced landslides.

\section{ACKNOWLEDGEMENT}

The analysis and write-up were carried out as a part of the first author's PhD studies at Faculty of Electronics and Telecommunication, VNU, Hanoi - University of Engineering and Technology. 


\section{REFERENCES}

[1] Ramesh MV. Real-time wireless sensor network for landslide detection. Sensor Technologies and Applications, 2009 SENSORCOMM'09 Third International Conference on; 2009: IEEE.

[2] Fosalau C, Zet C, Petrisor D. Implementation of a landslide monitoring system as a wireless sensor network. Ubiquitous Computing, Electronics \& Mobile Communication Conference (UEMCON), IEEE Annual; 2016: IEEE.

[3] Chang DTT, Tsai Y-S, Yang K-C. Study of Real-Time Slope Stability Monitoring System Using Wireless Sensor Network (WSN). Indonesian Journal of Electrical Engineering and Computer Science. 2013;11(3):1478-1488.

[4] Gian QA, Nguyen DC, Tran DN, Tran D-T. Monitoring of Landslides in Mountainous Regions based on FEM Modelling and Rain Gauge Measurements. International Journal of Electrical and Computer Engineering (IJECE). 2016;6(5):2106-2113.

[5] Rawat P, Singh KD, Chaouchi H, Bonnin JM. Wireless sensor networks: a survey on recent developments and potential synergies. The Journal of supercomputing. 2014;68(1):1-48.

[6] Nguyen D-C, Duc-Tan T, Tran D-N. Application of compressed sensing in effective power consumption of WSN for landslide scenario. Multimedia and Broadcasting (APMediaCast), 2015 Asia Pacific Conference on; 2015: IEEE.

[7] You L, Han Y, Li S, Su X. Soure and Transmission Control for Wireless Visual Sensor Networks with Compressive Sensing and Energy Harvesting. Indonesian Journal of Electrical Engineering and Computer Science. 2013;11(5):2468-2474.

[8] Raghunathan V, Schurgers C, Park S, Srivastava MB. Energy-aware wireless microsensor networks. IEEE Signal processing magazine. 2002;19(2):40-50.

[9] Zet C, Fosalau C, Petrişor D, Hogaş I. Study of the power consumtion of a landslide sensor node. Ubiquitous Computing, Electronics \& Mobile Communication Conference (UEMCON), IEEE Annual; 2016: IEEE.

[10] Pantazis NA, Nikolidakis SA, Vergados DD. Energy-efficient routing protocols in wireless sensor networks: A survey. IEEE Communications surveys \& tutorials. 2013;15(2):551-591.

[11] Nguyen CD, Tran TD, Tran ND, Huynh TH, Nguyen DT. Flexible and efficient wireless sensor networks for detecting rainfall-induced landslides. International Journal of Distributed Sensor Networks. 2015;2015.

[12] Buratti C, Conti A, Dardari D, Verdone R. An overview on wireless sensor networks technology and evolution. Sensors. 2009;9(9):6869-6896.

[13] Yu X, Wu P, Han W, Zhang Z. A survey on wireless sensor network infrastructure for agriculture. Computer Standards \& Interfaces. 2013;35(1):59-64.

[14] Castillo-Effer M, Quintela DH, Moreno W, Jordan R, Westhoff W. Wireless sensor networks for flash-flood alerting. Devices, Circuits and Systems, 2004 Proceedings of the Fifth IEEE International Caracas Conference on; 2004: IEEE.

[15] Kerkez B, Glaser SD, Bales RC, Meadows MW. Design and performance of a wireless sensor network for catchmentscale snow and soil moisture measurements. Water Resources Research. 2012;48(9).

[16] Chintalapudi K, Fu T, Paek J, Kothari N, Rangwala S, Caffrey J, Govindan R, Johnson E, Masri S. Monitoring civil structures with a wireless sensor network. IEEE Internet Computing. 2006;10(2):26-34.

[17] Li M, Liu Y. Underground structure monitoring with wireless sensor networks. Proceedings of the 6th international conference on Information processing in sensor networks; 2007: ACM.

[18] Li M, Liu Y. Underground coal mine monitoring with wireless sensor networks. ACM Transactions on Sensor Networks (TOSN). 2009;5(2):10.

[19] Ramesh MV. Design, development, and deployment of a wireless sensor network for detection of landslides. Ad Hoc Networks. 2014;13:2-18.

[20] Sheth A, Tejaswi K, Mehta P, Parekh C, Bansal R, Merchant S, Singh T, Desai UB, Thekkath CA, Toyama K. Senslide: a sensor network based landslide prediction system. Proceedings of the 3rd international conference on Embedded networked sensor systems; 2005: ACM.

[21] Arnhardt C, Asch K, Azzam R, Bill R, Fernandez-Steeger T, Homfeld S, Kallash A, Niemeyer F, Ritter H, Toloczyki M. Sensor based Landslide Early Warning System-SLEWS. Development of a geoservice infrastructure as basis for early warning systems for landslides by integration of real-time sensors. Geotechnologien science report. 2007;10:7588.

[22] Intrieri E, Gigli G, Mugnai F, Fanti R, Casagli N. Design and implementation of a landslide early warning system. Engineering Geology. 2012;147-148:124-136.

[23] Akyildiz IF, Stuntebeck EP. Wireless underground sensor networks: Research challenges. Ad Hoc Networks. 2006;4(6):669-686.

[24] Hu X, Wang B, Ji H. A wireless sensor network-based structural health monitoring system for highway bridges. Computer-Aided Civil and Infrastructure Engineering. 2013;28(3):193-209.

[25] Srbinovska M, Gavrovski C, Dimcev V, Krkoleva A, Borozan V. Environmental parameters monitoring in precision agriculture using wireless sensor networks. Journal of Cleaner Production. 2015;88:297-307. 